\title{
ARTí́CUlO \\ Asociación Scurria-Lessonia, indicador de integridad ecológica en praderas explotadas de huiro negro Lessonia berteroana (ex L. nigrescens) en el norte de Chile
}

Scurria-Lessonia association as an indicator of ecological integrity in exploited kelp beds of Lessonia berteroana (ex L. nigrescens) in northern Chile

\section{J.M. Alonso Vega ${ }^{1,2 *}$, Cynthia M. Asorey ${ }^{1,2}$ y Nicole Piaget $^{1}$}

\begin{abstract}
'Departamento de Biología Marina, Facultad de Ciencias del Mar, Universidad Católica del Norte, Casilla 117, Coquimbo, Chile ${ }^{2}$ Programa de Doctorado en Biología y Ecología Aplicada, Universidad Católica del Norte, Casilla 117, Coquimbo, Chile. *avega @ucn.cl
\end{abstract}

\begin{abstract}
Scurria scurra is a patelogastropod inhabiting the stipes and holdfasts cavities of Lessonia nigrescens complex species forming a herbivore-kelp association highly specialized. It has been proposed that the exploitation of kelp beds of Lessonia berteroana in northern Chile is a disturbance that affects Scurria-Lessonia association such as ENSO (El Niño-Southern Oscillation) events. Considering the above, and the important ecological role that have kelps as foundation species, this study proposes the use of Scurria-Lessonia association as an ecological indicator to monitor the integrity of kelp beds of L. berteroana. To achieve this, the abundance and size structure of S. scurra in plants of L. berteroana was evaluated under 3 different types of conservation strategies of fishing resources: open access areas and management areas (with harvest), and marine protected areas (without harvest) in the Region of Atacama and Region of Coquimbo, Chile. In addition, we monitored the time of recovery post-harvest of Scurria-Lessonia association in an experimental site (Lagunillas, $30^{\circ} \mathrm{S}$ ). The results show that $\mathrm{S}$. scurra population in open access areas is dominated by juveniles in low abundances, a situation not detected in management areas and marine protected areas. Furthermore, the recovery of S. scurra post-harvest of Lessonia at the experimental site took more than 2 years ( 30 months). An indicator was proposed to validate the actions of management plan of brown algae for open access areas to fisheries. This ecological indicator is easy to observe, evaluate and disseminate among users of the productive chain of Chilean kelp resource.
\end{abstract}

Key words: Kelp fisheries, ecological indicators, marine conservation, northern Chile

\begin{abstract}
Resumen.- Scurria scurra es un patelogastrópodo que habita los estipes y cavidades de discos adhesivos del complejo de especies Lessonia nigrescens formando una asociación herbívoro-alga altamente especializada. En el norte de Chile, se ha propuesto que la explotación de praderas de Lessonia berteroana es una perturbación que afecta la asociación Scurria-Lessonia de manera similar que los eventos ENOS (EI Niño-Oscilación del Sur). Considerando lo anterior, y el importante rol ecológico que cumplen las algas pardas como especies fundacionales, este estudio propone el uso de la asociación Scurria-Lessonia como un indicador ecológico para monitorear la integridad de praderas explotadas de L. berteroana. Se evaluó la abundancia y estructura de talla de S. scurra en plantas de L. berteroana bajo 3 distintos tipos de estrategias de conservación de recursos pesqueros: áreas de libre acceso y áreas de manejo (con cosecha), y áreas marinas protegidas (sin cosecha) en Atacama y Coquimbo (26-32 $\left.{ }^{\circ} \mathrm{S}\right)$. Además, se monitoreo el tiempo de recuperación post cosecha de la asociación Scurria-Lessonia en un sitio experimental (Lagunillas, $30^{\circ} \mathrm{S}$ ). Los resultados muestran que en áreas de libre acceso la población de Scurria está dominada por juveniles en bajas abundancias, situación no detectada en áreas de manejo y áreas marinas protegidas. Más aún, la recuperación de S. scurra post cosecha de Lessonia en el sitio experimental demoró más de 2 años (30 meses). Se propone un indicador para validar las acciones del plan de manejo de algas pardas para áreas de libre acceso a la pesquería. Este indicador ecológico es fácil de observar, evaluar y difundir entre los usuarios de la cadena productiva del recurso huiro negro.
\end{abstract}

Palabras clave: Pesquería algas pardas, indicadores ecológicos, conservación marina, norte de Chile

\section{INTRODUCCIÓN}

La integridad ecológica es una manera práctica para establecer si un plan de manejo, permite la explotación sustentable de un recurso natural sin afectar la salud del ecosistema (Carignan \& Villard 2002). Esto se logra evaluando la efectividad de las acciones de manejo a través de indicadores ecológicos comprensibles y comunicables a la sociedad (Niemi \& McDonald 2001). Los indicadores ecológicos proporcionan una medida de la respuesta del ecosistema a la perturbación 
antrópica (e.g., cosecha), cuantificando la desviación respecto a la integridad ecológica de algún atributo medible del ecosistema (Isasi-Catalá 2011). En este contexto, uno de los atributos comúnmente utilizado como indicadores ecológicos son las especies de macroflora o macrofauna (Ellison et al. 2005). Entre las principales razones para usar las especies como indicadores ecológicos destaca la facilidad para identificarlas y medirlas, y el número relativamente amplio de especies con respuestas conocidas a las perturbaciones; pero también es importante el interés público que la especie puede alcanzar y el costo relativamente bajo que se requiere para implementar su monitoreo (Niemi \& McDonald 2004).

Una especie indicadora es un término general para referirse a los enfoques que usan algunas especies para indicar una condición o respuesta al estrés y que puede aplicarse a otras con requerimientos ecológicos similares (Carignan \& Villard 2002). Esta definición incluye a las especies paraguas, especies claves, especies emblemáticas, gremios, entre otras (IsasiCatalá 2011).
Especies fuertemente asociadas con algunas características particulares del hábitat también puede ser usadas como indicadores (Niemi \& McDonald 2001), como sucede con Scurria scurra (Lesson, 1830) (Patellogastropoda, Lottidae) y el huiro negro: Lessonia berteroana Montagne y Lessonia spicata (Suhr) Santelices (ex L. nigrescens) (Laminariales, Phaeophyceae) (Santelices et al. 1980, Muñoz \& Santelices 1989, Meynard 2014) (Fig. 1).

En el norte de Chile, S. scurra es un molusco herbívoro que habita exclusivamente sobre los estipes y/o en cavidades dentro de discos adhesivos de L. berteroana. En el centro y sur de Chile, en cambio, S. scurra está asociada a L. spicata (Suhr) Santelices, una especie críptica de L. berteroana (Tellier et al. 2011, Meynard 2014) (Fig. 1). Este patelogastrópodo también habita en discos adhesivos de Durvillaea antarctica y $L$. trabeculata, y rara vez en otros sustratos biogénicos (e.g., Perumytilus purpuratus) o sobre roca (Vásquez et al. 2001). La distribución latitudinal de S. scurra abarca la costa de Chile entre los $24^{\circ} \mathrm{S}$ y $42^{\circ} \mathrm{S}$, siempre en presencia del huiral que forma
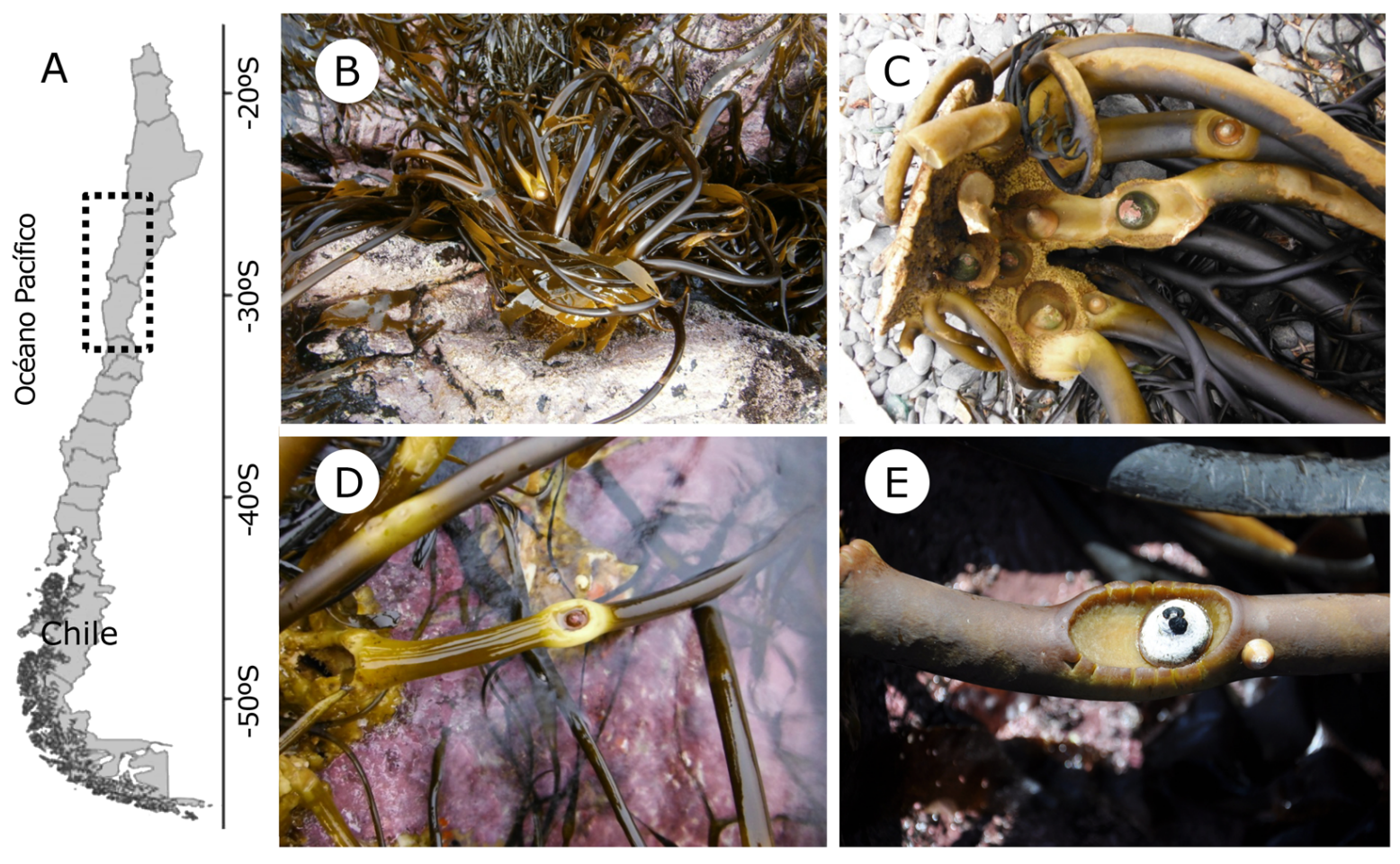

Figura 1. A) Área de estudio, destacada en el rectángulo con línea punteada; B) Asociación Scurria-Lessonia; C) Scurria scurra sobre estipes de Lessonia berteroana (ex L. nigrescens) en el área marina protegida de Isla Choros, D) en el área de libre acceso de Totoral Bajo; y E) sobre un estipe de Lessonia spicata (ex L. nigrescens) en la costa de Concepción / A) The study area highlighted in rectangle with dotted line; B) Scurria-Lessonia association; C) Scurria scurra on stipes of Lessonia berteroana (ex L. nigrescens) in marine protected areas of Isla Choros, D) in open access area of Totoral Bajo; and E) on a stipe of L. spicata in Concepción coast 
el complejo de especies L. nigrescens en la zona intermareal baja (Espoz et al. 2004); constituido por L. berteroana al norte de los $30^{\circ} \mathrm{S}$ y L. spicata hacia el sur de esta latitud, respectivamente (González et al. 2012). Los individuos más grandes de $S$. scurra habitan las partes proximales de los estipes en cavidades que horadan al alimentarse progresivamente del tejido de Lessonia spp. (Muñoz \& Santelices 1989, Camus et al. 2013) (Fig. 1), mientras que los individuos juveniles se refugian en las cavidades internas de los discos de adhesión (Cancino \& Santelices 1984, Vásquez \& Santelices 1984). Esta particular asociación entre Scurria y Lessonia ha sido explicada por la especialización que tiene $S$. scurra al hábitat y los recursos alimenticios que ofrece Lessonia (Muñoz \& Santelices 1989, Camus 1994, Meynard 2014).

Históricamente, eventos ENOS (El Niño-Oscilación del Sur) producen procesos de extinción local y recolonización de las praderas de $L$. berteroana en el norte de Chile afectando significativamente la asociación Scurria-Lessonia (Camus 1994, Espoz et al. 2004). El efecto neto de esta perturbación natural resulta en cambios de los patrones de distribución a nivel local y regional y en la abundancia de $S$. scurra, debido a la lenta tasa de renovación de las praderas de L. berteroana después del evento (Camus 1994), incluso se ha detectado una compresión del rango de distribución de la especie hacia el sur (Espoz et al. 2004).

Se ha postulado que la pesquería de algas pardas, una perturbación de origen antrópico, también amenaza la asociación Scurria-Lessonia (Espoz et al. 2004), y la biota que estas especies fundacionales sostienen (Camus 1994, Vega et al. 2014). Considerando lo anterior, y que S. Scurria es un Patelogastrópodo representativo de las comunidades asociadas a praderas del complejo de especies L. nigrescens (Cancino \& Santelices 1984, Vásquez \& Santelices 1984, Camus 1994, Vásquez et al. 2001) (Fig. 1), este estudio propone el uso de la asociación Scurria-Lessonia como un indicador ecológico para monitorear la integridad de las praderas explotadas de huiro negro L. berteroana (ex L. nigrescens) en el norte de Chile. Para lograr este objetivo, se comparó la abundancia y estructura de tallas de $S$. scurra en los estipes de plantas ubicadas en praderas afectas a 3 distintas estrategias de conservación (Vega et al. 2014). Complementariamente, se determinó el tiempo de recuperación de $S$. scurra en los estipes de plantas de L. berteroana después de una cosecha experimental (denudación). Con los resultados de ambos experimentos, se construyó un indicador ecológico para monitorear la integridad de praderas explotadas del recurso huiro negro Lessonia spp., particularmente en áreas de libre acceso a la pesquería de algas pardas.

\section{Materiales y MÉTODOS}

Se evalúo la abundancia y la estructura de tallas de individuos de $S$. scurra en estipes de plantas de L. berteroana ubicadas en áreas de libre acceso (ALA), áreas de manejo (AMERB) y áreas marinas protegidas (AMP) en la Región de Atacama y Región de Coquimbo, entre noviembre y diciembre del 2013 (Fig. 1). Las localidades muestreadas fueron: Totoral Bajo $\left(27,757^{\circ} \mathrm{S}-71,064^{\circ} \mathrm{W}\right)$, Caleta Angosta $\left(28,250^{\circ} \mathrm{S}\right.$ $\left.71,163^{\circ} \mathrm{W}\right)$, Lagunillas $\left(30,103^{\circ} \mathrm{S}-71,383^{\circ} \mathrm{W}\right)$ y Talquilla $\left(30,864^{\circ} \mathrm{S}-71,683^{\circ} \mathrm{W}\right)$, donde hay áreas de manejo y áreas de libre acceso adyacentes (Vega et al. 2014). En ALA, cualquier pescador que tenga inscrito el recurso huiro negro (Lessonia spp.) puede realizar cosecha, en cambio en AMERB, sólo realizan cosecha aquellos pescadores que forman parte de un gremio. Además, se muestrearon individuos de $S$. scurra en plantas de L. berteroana en AMP Isla Grande de Atacama $\left(27,248^{\circ} \mathrm{S}-70,974^{\circ} \mathrm{W}\right)$ e Isla Choros $\left(29,259^{\circ} \mathrm{S}-71,535^{\circ} \mathrm{W}\right)$, donde la cosecha de Lessonia está prohibida (Vega et al. 2014).

La frecuencia de ocurrencia de S. scurra por planta se estimó detectando la presencia o ausencia de patelogastrópodos en plantas de Lessonia ubicadas dentro de 9 cuadrantes de $1 \mathrm{~m}^{2}$ (Vega et al. 2014). La frecuencia de ocurrencia de $S$. scurra en estipes se evalúo muestreando aleatoriamente 20 plantas adultas de Lessonia $(\geq 20 \mathrm{~cm}$ diámetro del disco de adhesión; Vásquez et al. 2012). El tamaño de los individuos de $S$. scurra fue obtenido in situ midiendo la base de la concha cónica con un vernier digital.

La abundancia de S. scurra en plantas de L. berteroana fue monitoreada temporalmente en un sitio experimental de denudación en Lagunillas (Oróstica et al. 2014, Rodríguez et al. 2014). La población de $S$. scurra fue muestreada al inicio, en marzo 2011, cuando se efectúo la cosecha de plantas en el transecto experimental (control), y posteriormente a 8, 20 y 30 meses después de la cosecha. En cada muestreo, se evaluó el número de $S$. scurra en estipes de 20 plantas; y en cada observación, se midió in situ el diámetro de la concha del individuo y el correspondiente diámetro del estipe de Lessonia con un vernier digital.

La frecuencia de ocurrencia y abundancia de $S$. scurra en estipes y plantas de Lessonia fue comparada entre las distintas estrategias de conservación (ALA, AMERB y AMP) usando análisis de varianza (ANDEVA), considerando a la pradera de L. berteroana como un factor anidado (Tabla 1). La frecuencia de ocurrencia y abundancia de $S$. scurra en estipes y plantas de Lessonia en el sitio experimental fue comparada entre muestreos $(0,8,20$ y 30 meses) utilizando ANDEVA de un factor. El incremento del tamaño de la concha de $S$. scurra yel 
Tabla 1. ANDEVA usando la estrategia de conservación como factor principal (Tipo) y pradera de Lessonia berteroana (Sitio) como factor anidado para evaluar la hipótesis que la asociación Scurria-Lessonia es modificada en áreas de libre acceso (ALA: Área de Libre Acceso, AMERB: Área de Manejo, AM P: Área Marina Protegida) / ANOVA using the conservation strategy as main factor (Type) and Lessonia berteroana bed (Site) as nested factor to evaluate the hypothesis that Scurria-Lessonia association is modified in open access areas (ALA: Open Access Area, AMERB: Management Area, AMP: Marine Protected Area)

\begin{tabular}{clccccc}
\hline \multicolumn{1}{c}{ S. scurra } & Factor & GL & CM & Valor F & Valor $P$ & Prueba de Tukey \\
\hline en \% por plantas en la pradera & Tipo & 2 & 1,656 & 66,1 & 0,0001 & AMP $>$ AMERB $>$ ALA \\
& Sitio (Tipo) & 7 & 0,009 & 0,356 & 0,925 & \\
& Error & 80 & 0,025 & & & \\
en $\mathrm{n}^{\circ}$ de individuos por planta & Tipo & 2 & 950,4 & 55,8 & 0,0001 & AMP $>$ AMERB $>$ ALA \\
& Sitio (Tipo) & 7 & 7,602 & 0,446 & 0,872 & \\
& Error & 190 & 17,029 & & & \\
en \% por estipe & Tipo & 2 & 0,133 & 52,2 & 0,0001 & AMP $>$ AMERB $>$ ALA \\
& Sitio (Tipo) & 7 & 0,002 & 0,882 & 0,521 & \\
& Error & 190 & 0,003 & & & \\
\hline
\end{tabular}

\begin{abstract}
Tabla 2. Indicadores ecológicos basados en la asociación Scurria-Lessonia para monitorear la integridad de praderas explotadas de Lessonia berteroana (DS: desviación estándar; $\varnothing$ : diámetro de la concha de Scurria scurra) / Ecological Indicators based in Scurria-Lessonia association to monitor the integrity of exploited kelp beds of Lessonia berteroana (DS: standard deviation; $\varnothing$ : shell diameter of Scurria scurra)
\end{abstract}

\begin{tabular}{lccc}
\hline \multirow{2}{*}{$\begin{array}{l}\text { Asociación } \\
\text { Scurria-Lessonia }\end{array}$} & $\begin{array}{c}\text { Pradera } \\
\text { no explotada }\end{array}$ & $\begin{array}{c}\text { Cosecha } \\
\text { sustentable }\end{array}$ & $\begin{array}{c}\text { Alta presión } \\
\text { de cosecha }\end{array}$ \\
\cline { 3 - 4 } $\begin{array}{l}\text { Frecuencia de ocurrencia } \\
\text { por planta }\end{array}$ & $\begin{array}{c}\text { Muy frecuente } \\
(>70 \%)\end{array}$ & $\begin{array}{c}\text { Frecuente } \\
(30-70 \%)\end{array}$ & $\begin{array}{c}\text { Poco Frecuente } \\
(<30 \%)\end{array}$ \\
$\begin{array}{l}\text { Frecuencia de ocurrencia } \\
\text { por estipes }\end{array}$ & $\begin{array}{c}\text { Muy frecuente } \\
(>25 \%)\end{array}$ & $\begin{array}{c}\text { Frecuente } \\
(15-25 \%)\end{array}$ & $\begin{array}{c}\text { Poco Frecuente } \\
(<15 \%)\end{array}$ \\
$\begin{array}{l}\text { Tamaño promedio } \\
\text { (media } \pm \text { DS; } \mathrm{n}=250)\end{array}$ & $\begin{array}{c}\text { Optimo } \\
(2,2 \pm 0,76)\end{array}$ & $\begin{array}{c}\text { Normal } \\
(1,8 \pm 0,58)\end{array}$ & $\begin{array}{c}\text { Bajo Talla } \\
(1,5 \pm 0,57)\end{array}$ \\
$\begin{array}{l}\text { Individuos pequeños } \\
(<1,5 \text { cm Ø concha) }\end{array}$ & $\begin{array}{c}\text { Poco frecuente } \\
(<20 \%)\end{array}$ & $\begin{array}{c}\text { Frecuente } \\
(20-30 \%)\end{array}$ & $\begin{array}{c}\text { Muy Frecuente } \\
(>30 \%)\end{array}$ \\
$\begin{array}{l}\text { Individuos grandes } \\
(>2,5 \text { cm Ø concha })\end{array}$ & $\begin{array}{c}\text { Muy frecuente } \\
(>15 \%)\end{array}$ & $\begin{array}{c}\text { Frecuente } \\
(5-15 \%)\end{array}$ & $\begin{array}{c}\text { Poco Frecuente } \\
(<5 \%)\end{array}$ \\
\hline $\begin{array}{l}\text { Plan de Manejo } \\
(\text { PM })\end{array}$ & $\begin{array}{c}\text { Pradera } \\
\text { Referencial }\end{array}$ & $\begin{array}{c}\text { Acciones del PM son } \\
\text { adecuadas }\end{array}$ & $\begin{array}{c}\text { Acciones del PM deben } \\
\text { ser revisadas y } \\
\text { corregidas o modificadas }\end{array}$ \\
\hline
\end{tabular}

diámetro del estipe de $L$. berteroana en el tiempo $(0,8,20$ y 30 meses) fue evaluado a través de análisis de covarianza (ANCOVA). La distribución normal y homocedasticidad de la varianza fue verificada con la prueba de Barlett y prueba de Lilliefors, mientras que los datos porcentuales fueron transformados al arcoseno de la raíz cuadrada antes de realizar los análisis estadísticos. Cuando el ANDEVA o ANCOVA presentó diferencias significativas se aplicó la prueba a posteriori de Tukey (Quinn \& Keough 2002).

\section{Resultados}

La frecuencia de ocurrencia y abundancia de $S$. scurra en los estipes y plantas de L. berteroana depende del tipo de estrategia de conservación al que está sometida la pradera (Fig. 2, Tabla 1). Scurria scurra fue significativamente más frecuente en los estipes de plantas ubicadas en praderas de AMP que de AMERB o ALA (Tabla 1), pero es en praderas de ALA donde la disminución de $S$. scurra en las plantas de L. berteroana fue 
significativamente más notoria (Figs. 2A y B, Tabla 1). En general, la población de $S$. scurra de ALA estuvo dominada por juveniles, mientras que en AMERB y AMP fueron más frecuentes las clases de tallas superiores (Fig. 2C). Los individuos de mayor tamaño de $S$. scurra se detectaron en las plantas ubicadas en AMP (Fig. 2C).

En el sitio experimental de cosecha, el reclutamiento de $S$. scurra sobre estipes aumentó a medida que el tamaño del alga es mayor (ANDEVA $F_{(3,46)}: 16,502, P<0,01 ;$ Fig. 3). Luego de 30 meses de realizada la cosecha experimental (2,5 años), la densidad promedio de $S$. scurra por planta alcanzó niveles semejantes al encontrado al inicio del experimento (Fig. 3A). El incremento del tamaño de $S$. scurra en el tiempo covaría significativamente con el diámetro de los estipes de las plantas de L. berteroana (ANCOVA $F_{(3,298)}: 7,3211, P<0,01$; Fig. 3B). Así, la progresión temporal de la estructura de tallas de la población de $S$. scurra ocurre a medida que las plantas de $L$. berteroana crecen y renuevan la pradera (Fig. 3C). Sin embargo, sólo el $20 \%$ de la población de S. scurra logra tamaños en las clases superiores de la estructura de tallas semejante al observado al inicio del experimento.
La frecuencia de ocurrencia, la abundancia y el tamaño de S. scurra son atributos demográficos útiles para construir los indicadores ecológicos basados en la asociación ScurriaLessonia (Tabla 2). El punto de referencia objetivo del indicador Scurria-Lessonia, que representa el estado de conservación deseado del recurso huiro negro L. berteroana, está dado por los valores de los descriptores demográficos de $S$. scurra encontrados en AMP; mientras que el punto de referencia límite del indicador está dado por los valores de los descriptores demográficos de S. scurria detectados en AMERB. Así, cuando los valores están por sobre el punto de referencia límite, la explotación del recurso huiro negro $L$. berteroana es sustentable, y las acciones del plan de manejo son adecuadas (e.g., AMERB); pero cuando los valores están por debajo del punto de referencia límite, por ejemplo en ALA, se constituye un estado de conservación no deseado, y las acciones del plan de manejo implementadas para el recurso deben ser revisadas, corregidas o modificadas (Tabla 2).
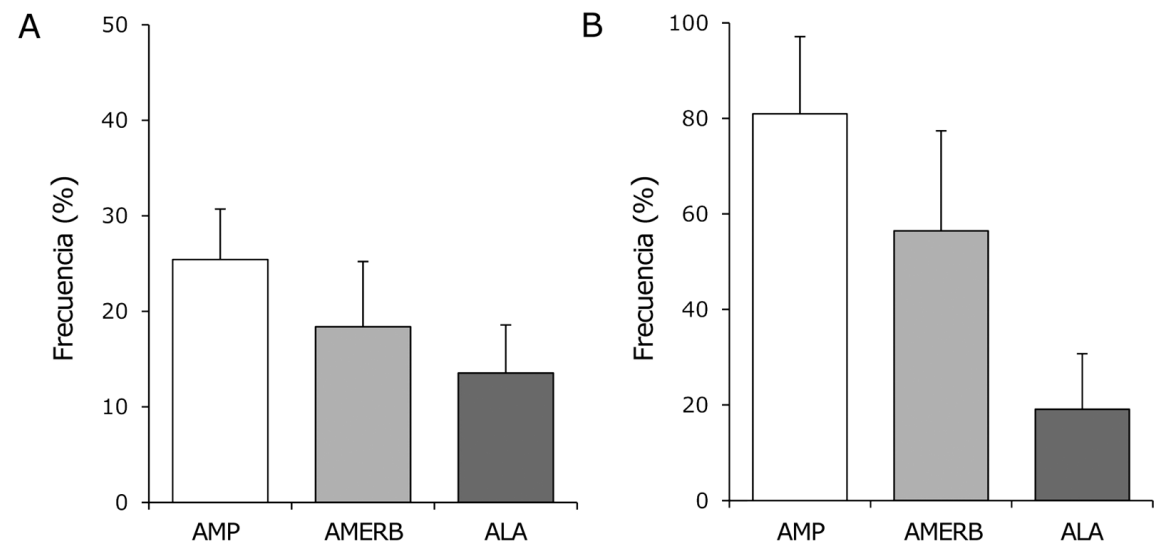

C

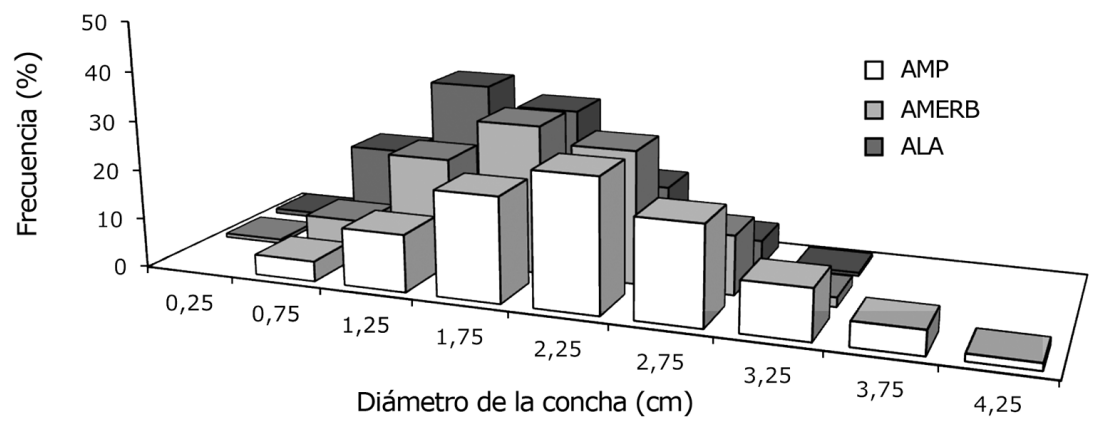

Figura 2. A) Frecuencia de ocurrencia de Scurria scurra en estipes; B) en plantas de Lessonia berteroana; y (C) estructura de tallas de la población de Scurria scurra en praderas de Lessonia con distintas estrategias de conservación (AM P: Área Marina Protegida; AM ERB: Área de Manejo; ALA: Área de Libre Acceso) / A) Frequency of occurrence of Scurria scurra in stipes; B) plants of Lessonia berteroana; and (C) population's size structure of Scurria scurra in kelp beds of Lessonia with different conservation strategies (AMP: Marine Protected Area; AMERB: Management Area; ALA: Open Access Area) 

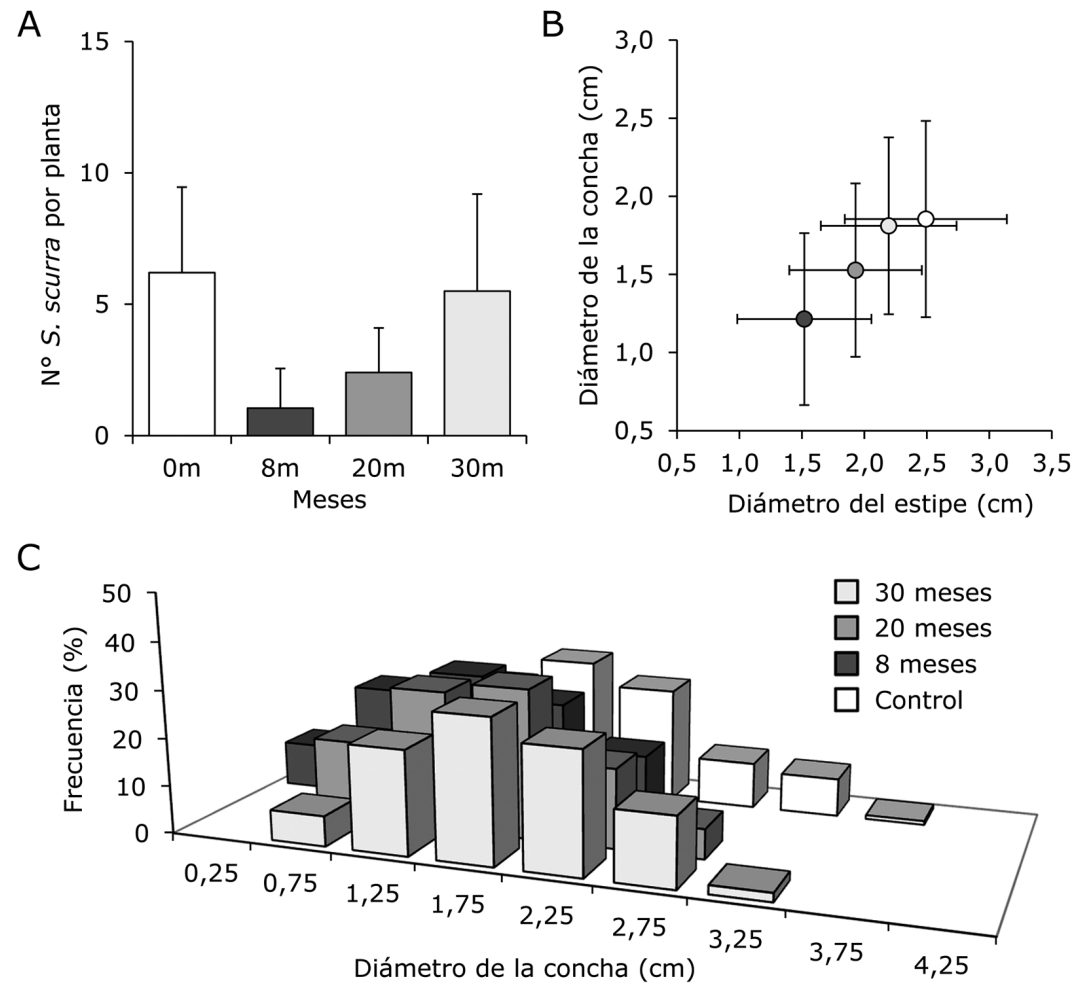

Figura 3. A) Variación temporal de la abundancia de Scurria scurra en plantas de Lessonia berteroana; B) relación entre el tamaño de Scurria scurra y el diámetro del estipe de Lessonia berteroana; y C) estructura de tallas de la población de Scurria scurra en la pradera de Lessonia en el sitio experimental / A) Temporal variation of Scurria scurra in plants of Lessonia berteroana; B) relationship between the size of Scurria scurra and stipe diameter of Lessonia berteroana; and C) size structure of Scurria scurra population in kelp beds of Lessonia at experimental site

\section{DiscuSIón}

La explotación de L. berteroana en el norte de Chile tiene un efecto negativo en la asociación Scurria-Lessonia, particularmente en áreas de libre acceso a la pesquería de algas pardas. La alta presión de cosecha ejercida en praderas de Lessonia sujetas a este tipo de estrategia de conservación (Vega et al. 2014), se ve reflejada en una baja abundancia de individuos de S. scurra en los estipes de las plantas y una estructura de tallas dominada por juveniles. Dentro de áreas marinas protegidas, donde no se explota Lessonia, los individuos adultos de este patelogastrópodo son frecuentes y abundantes sobre las plantas, tal como fue descrito antes del comienzo de la explotación intensiva del recurso huiro negro en el 2000 (Santelices et al. 1980, Muñoz \& Santelices 1989, Camus 1994). En áreas de manejo donde la explotación de recursos bentónicos es realizada a través de co-manejo (Quiñones \& Aliaga 2004), la asociación Scurria-Lessonia persiste, porque las acciones de manejo propenden a buenas prácticas de cosecha (Vásquez et al. 2012), manteniendo la integridad ecológica de las poblaciones de L. berteroana. En este contexto, las áreas de manejo contribuyen a la conservación de funciones y servicios ecosistémicos que proveen las praderas de Lessonia, que en áreas de libre acceso a la pesquería de algas pardas están en riesgo permanente (ver ejemplos en Almanza et al. 2012, Almanza \& Buschmann 2013 para Macrocystis pyrifera; Molina et al. 2014 para Lessonia trabeculata).

En el extremo norte de Chile $\left(18^{\circ} \mathrm{S}\right.$ a $\left.23^{\circ} \mathrm{S}\right)$, el evento ENOS es una perturbación que ocasiona procesos de extinción y recolonización local de Lessonia y $S$. scurra correlacionados con la latitud (Camus 1994, Espoz et al. 2004). Sin embargo, la recolonización de $S$. scurra no está necesariamente acoplada con Lessonia (Meynard 2014). Esta pérdida temporal del patelogastrópodo asociado en algunas praderas contribuye a la dinámica geográfica de las poblaciones de Lessonia (Camus 1994). Más aún, también hay una perdida potencial de funciones, como por ejemplo, el rol compensatorio que cumple S. scurra en las poblaciones de Lessonia spp. (Aguilera 2011), al reducir el riesgo de desprendimiento y aumentar la expectativa 
de vida a través de la 'poda' de los estipes más viejos de las plantas adultas (Santelices et. al. 1980, Muñoz \& Santelices 1989). La extracción activa del recurso huiro negro $L$. berteroana es una perturbación con efectos ecológicos potencialmente equivalente a los producidos por un evento ENOS, a escala local. Sin embargo, en una perspectiva pesquera dichos efectos pueden ser identificados en el plan de manejo y mitigados con acciones dirigidas a conservar el recurso huiro negro y la integridad ecológica de las praderas de Lessonia (Camus 1994, Vásquez et al. 2012, Vega et al. 2014).

La capacidad reproductiva y la diversidad genética son atributos de $S$. scurra que deben ser resguardados en las praderas explotadas de Lessonia. Tanto Lessonia como $S$. scurra tienen un bajo potencial de dispersión, poblaciones genéticamente estructuradas y quiebres filogeográficos entre los $27^{\circ} \mathrm{S}$ y $30^{\circ} \mathrm{S}$ (Tellier et al. 2011, Haye et al. 2014, Meynard 2014). Estos antecedentes sugieren una historia biogeográfica común en el norte de Chile, y sustentan la idea que $S$. scurra es un herbívoro altamente especializado (Meynard 2014), muy sensible a la explotación de las praderas de Lessonia (Camus 1994, Espoz et al. 2004). A pesar de esto, se conoce muy poco sobre el ciclo de vida de S. scurra. En otras especies de patelogastrópodos (e.g., Patella ferruginea, P. granularis), la fecundidad eíndice gonadosomático se correlaciona con el tamaño, de manera que hembras más grandes contribuyen más al pool larval (Espinosa et al. 2006, Vat 2000), y es esperable que esto también suceda en S. scurra (Meynard 2014). A diferencia de lo que ocurre en California donde la explotación de Lottia gigantea no afecta la diversidad genética porque las poblaciones no están genéticamente estructuradas (Fenberg $e t$ al. 2010), S. scurra presenta poblaciones genéticamente estructuradas con un flujo génico limitado hacia ambos lados del quiebre filogeográfico reportado en la literatura $\left(30^{\circ} \mathrm{S}\right.$ en Haye et al. 2014; $27^{\circ} \mathrm{S}$ en Meynard 2014). Así, la abundancia y el tamaño de $S$. scurra además de ser un indicador útil para monitorear la integridad ecológica de praderas explotadas de Lessonia, también puede ser usada como una estrategia para la preservación de este herbívoro clave del intermareal rocoso chileno (Espoz et al. 2004).

La abundancia y el tamaño de $S$. scurra en los estipes de las plantas pueden ser utilizados comoun indicador de integridad ecológica en las praderas explotadas de L. berteroana, y como un insumo o factor de decisión al evaluar las acciones establecidas en el plan de manejo para el recurso huiro negro (Tabla 2). Así, cuando los patelogastrópodos en los estipes de las plantas de Lessonia son en su mayoría pequeños (juveniles), poco frecuentes, y tienen una baja abundancia, entonces la pradera está sometida a una alta presión de cosecha (Vega et al. 2014), lo cual fue observado en áreas de libre acceso. En este escenario, hay un riesgo potencial de pérdida de funciones y servicios ecosistémicos en la pradera de Lessonia, por lo que la cosecha debe ser detenida y las acciones de manejo deben ser revisadas y corregidas o modificadas. En cambio, cuando los individuos de $S$. scurra son grandes (adultos), frecuentes y abundantes en los estipes de las plantas, se reconoce una pradera de Lessonia poco intervenida, natural e integra. En el contexto del manejo de recursos lo anterior puede ser una meta a lograr (Niemi \& MacDonald 2004, Carignan \& Villard 2002). No obstante, también existen situaciones intermedias como las detectadas en las áreas de manejo, donde el estado de la población de $S$. scurra sugiere una explotación sustentable que involucran acciones de manejo que propenden a la conservación de las praderas de Lessonia. Finalmente, se debe destacar que las áreas marinas protegidas contienen praderas de Lessonia referenciales que permiten discriminar efectos generados por otro tipo de perturbaciones (e.g., evento ENOS; Vásquez et al. 2001) que pueden interactuar con la pesquería de algas pardas (Camus 1994, Vega et al. 2014), y requerir de acciones complementarias al plan de manejo implementado para el recurso.

La asociación Scurria-Lessonia es un indicador útil para monitorear la integridad ecológica de praderas explotadas de L. berteroana porque: (a) la morfología de la concha de $S$. scurra es fácil de reconocer y de medir en terreno; (b) algunas respuestas demográficas (e.g., densidad, estructura de tallas) a las perturbaciones son relativamente conocidas (Muñoz \& Santelices 1989, Camus 1994, Espoz 2004, Meynard 2014); y (c) es una especie clave en la persistencia de las poblaciones de Lessonia (Aguilera 2011, Isasi-Catalá 2011). No obstante, todavía se requiere investigar diversos aspectos de la biología y ecología de este patelogastrópodo que podrían potenciar al indicador ecológico. Adicionalmente, la implementación de este indicador requiere de un presupuesto mínimo, y el seguimiento puede ser ejecutado por los propios pescadores ó por cualquier persona interesada en realizar el monitoreo, después de un breve entrenamiento básico.

Lessonia berteroana es una de las especies crípticas que conforman el complejo $L$. nigrescens, la otra es $L$. spicata (González et al. 2012). Aunque difieren en distintos rasgos ecofisiológicos (Tellier et al. 2011), la asociación ScurriaLessonia ocurre en las dos especies debido a la alta similitud morfológica de las plantas (Meynard 2014) (Fig. 1). Así, los indicadores ecológicos propuesto para L. berteroana también pueden ser usado para establecer la integridad ecológica de praderas explotadas de $L$. spicata en la zona centro-sur de Chile (e.g., Talquilla, al sur de $\operatorname{los} 30^{\circ} \mathrm{S}$ ). No obstante, se requieren estudios comparativos de la asociación ScurriaLessonia entre ambas especies crípticas de Lessonia. 
En conclusión, la asociación Scurria-Lessonia puede ser usada como un indicador para monitorear el efecto de las acciones del plan de manejo en la integridad ecológica de las praderas explotadas del huiro negro $L$. berteroana (ex $L$. nigrescens). Además, este indicador ecológico es fácil de observar, evaluar y difundir y/o aplicar por los propios usuarios de la cadena productiva de este recurso pesquero bentónico.

\section{Agradecimientos}

JMAV y CMA agradecen a la Universidad Católica del Norte (UCN) y a la Comisión Nacional de Investigación Científica y Tecnológica (CONICYT, Beca N 21110630 y N² 21120351 , respectivamente), la beca de estudio en el Programa de Doctorado en Biología y Ecología Aplicada, UCN (Coquimbo, Chile). JMAV agradece el financiamiento de la Subsecretaria de Pesca y Acuicultura (Proyectos: ID N 4728-132-LE13, ID N $\left.{ }^{\circ} 4728-133-L E 13\right)$ y a los equipos de trabajo de ECOS y Centro de Estudios de Sistemas Sociales-CESSO. Se agradece a E. Macaya la fotografía de la asociación ScurriaLessonia en Concepción. También se agradecen los comentarios y correcciones al manuscrito de tres evaluadores anónimos.

\section{LITERATURA CITADA}

Aguilera MA. 2011. The functional roles of herbivores in the rocky intertidal systems in Chile: A review of food preferences and consumptive effects. Revista Chilena de Historia Natural 84:241-261.

Almanza V \& AH Buschmann. 2013. The ecological importance of Macrocystis pyrifera (Phaeophyta) forests towards a sustainable management and exploitation of Chilean coastal benthic co-management areas. International Journal of Environment and Sustainable Development 12(4): 341-360.

Almanza V, AH Buschmann, MC Hernández-González \& LA Henríquez. 2012. Can giant kelp (Macrocystis pyrifera) forests enhance invertebrate recruitment in southern Chile? Marine Biology Research 8(9): 855-864.

Camus PA. 1994. Dinámica geográfica en las poblaciones de Lessonia nigrescens Bory (Pheophyta) en el norte de Chile: importancia de la extinción local durante eventos El Niño de gran intensidad. Revista de Investigaciones Científicas y Tecnológicas, Serie Ciencias del Mar, Chile 3: 58-70.

Camus PA, PA Arancibia \& I Ávila-Thieme. 2013. Una caracterización trófica de los consumidores intermareales en las costas rocosas de Chile. Revista de Biología Marina y Oceanografía 48(3): 431-450.

Cancino J \& B Santelices. 1984. Importancia ecológica de los discos adhesivos de Lessonia nigrescens Bory (Phaeophyta) en Chile central. Revista Chilena de Historia Natural 57: 23-33.
Carignan V \& MA Villard. 2002. Selecting indicators species to monitor ecological integrity: a review. Environmental Monitoring and Assessment 78: 45-61.

Ellison AM, MS Bank, BD Clinton, EA Colburn, K Elliott, CR Ford, DR Foster, BD Kloeppel, JD Knoepp, GM Lovett, J Mohan, DA Orwig, NL Rodenshouse, WV Sobczak, KA Stinson, JK Stone, CM Swan, J Thompson, B Von Holle \& JR Webster. 2005. Loss of foundation species: consequences for the structure and dynamics of forested ecosystems. Frontiers in Ecology and the Environment 3: 479-486.

Espinosa F, JM Guerra-García, D Fa \& JC Garcýa-Gómez. 2006. Aspects of reproduction and their implications for the conservation of the endangered limpet, Patella ferruginea. Invertebrate Reproduction and Development 49(1-2): 85-92.

Espoz C, DR Lindberg, JC Castilla \& WB Simison. 2004. Los patelogastrópodos intermareales de Chile y Perú. Revista Chilena de Historia Natural 77(2): 257-283.

Fenberg PB, ME Hellberg, L Mullen \& K Roy. 2010. Genetic diversity and population structure of the sizeselectively harvested owl limpet, Lottia gigantea. Marine Ecology 31(4): 574-583.

González A, J Beltrán, L Hiriart-Bertrand, V Flores, B de Reviers, JA Correa \& B Santelices. 2012. Identification of cryptic species in the Lessonia nigrescens complex (Phaeophyceae, Laminariales). Journal of Phycology 48(5):1153-1165.

Haye PA, NI Segovia, NC Muñoz-Herrera, FE Gálvez, A Martínez, A Meynard, MC Pardo-Gandarillas, E Poulin \& S Faugeron. 2014. Phylogeographic structure in benthic marine invertebrates of the southeast Pacific coast of Chile with differing dispersal potential. PloS One 9(2), e88613.

Isasi-Catalá E. 2011. Los conceptos de especies indicadoras, paraguas, banderas y claves: su uso y abuso en ecología de la conservación. Interciencia 36(1): 31-38.

Meynard A. 2014. Grado de especialización ecológica y filogeografía comparada entre el herbívoro especialista Scurria scurra y sus dos algas hospederos en la costa chilena. Tesis Doctoral, Facultad de Ciencias Biológicas, Pontificia Universidad Católica de Chile, Santiago, 135 pp.

Molina P, FP Ojeda, M Aldana, VM Pulgar, MR GarcíaHuidobro \& J Pulgar. 2014. Spatial and temporal variability in subtidal macroinvertebrates diversity patterns in a management and exploitation area for benthic resources (MEABRs). Ocean and Coastal Management 93: 121-128.

Muñoz M \& B Santelices. 1989. Determination of the distribution and abundance of the limpet Scurria scurra on the stipes of the kelp Lessonia nigrescens in central Chile. Marine Ecology Progress Series 54: 277-285.

Niemi GJ \& ME McDonald. 2004. Application of ecological indicators. Annual Review of Ecology, Evolution, and Systematics 35: 89-111. 
Oróstica MH, MA Aguilera, GA Donoso, JA Vásquez \& BR Broitman. 2014. Effect of grazing on distribution and recovery of harvested stands of Lessonia berteroana kelp in northern Chile. Marine Ecology Progress Series 511: 71-82.

Quinn GP \& MJ Keough. 2002. Experimental design and data analysis for biologists, 537 pp. Cambridge University Press, Cambridge.

Quiñones RA \& B Aliaga. 2004. Conceptos fundamentales de administración pesquera. En: Werlinger C (ed). Biología marina y oceanografía: Conceptos y procesos, pp. 595-618. Trama Impresores, Concepción.

Rodríguez D, MH Oróstica \& JA Vásquez. 2014. Coalescence in wild organisms of the intertidal population of Lessonia berteroana in northern Chile: management and sustainability effects. Journal of Applied Phycology 26(2): 1115-1122.

Santelices B, JC Castilla, J Cancino \& P Schmiede. 1980. Comparative ecology of Lessonia nigrescens and Durvillaea antarctica (Phaeophyta) in central Chile. Marine Biology 59(2): 119-132.

Tellier F, JMA Vega, BR Broitman, JA Vasquez, M Valero \& S Faugeron. 2011. The importance of having two species instead of one in kelp management: the Lessonia nigrescens species complex. Cahiers de Biologie Marine 52(4): 455-465.
Vásquez JA \& B Santelices. 1984. Comunidades de macroinvertebrados en discos adhesivos de Lessonia nigrescens Bory (Phaeophyta) en Chile central. Revista Chilena de Historia Natural 57: 131-154.

Vásquez JA, D Veliz \& LM Pardo. 2001. Biodiversidad de macroinvertebrados bajo las grandes algas. En: Alveal K \& T Antezana (eds). Sustentabilidad de la biodiversidad. Un problema actual: Bases científico técnicas, teorizaciones y proyecciones, pp. 293-308. Universidad de Concepción, Concepción.

Vat LS. 2000. The growth and reproduction of Patella granularis (Mollusca: Patellogastropoda) on the south-east coast of South Africa. Thesis Doctoral, Faculty of Science, Rhodes University, Grahamstown, 247 pp.

Vega JMA, BR Broitman \& JA Vásquez. 2014. Monitoring the sustainability of Lessonia nigrescens (Laminariales, Phaeophyceae) in northern Chile under strong harvest pressure. Journal of Applied Phycology 26(2): 791-801. 